\title{
RESULTS FROM A FERMILAB NEUTRINO BEAM DUMP EXPERIMENT
}

R.C. Ball, C.T. Coffin, H.R. Gustafson, L.W. Jones, M.J. Longo, T.J. Robertsa, B.P. Roe, E. Wang

University of Michigan, Ann Arbor, MI 48109

$$
\begin{aligned}
& \text { C. Castoldi, G. Conforto } \\
& \text { INFN, Florence, Italy }
\end{aligned}
$$

M.B. Crisler, J.S. Hoftun, T.Y. Ling, T.A. Romanowski, J.T. Volk Ohio State University, Columbus, $\mathrm{OH} 43210$

\author{
S. Childressb \\ University of Washington, Seattle, WA 98195 \\ M.E. Duffy, G.K. Fanourakis, R.J. Loveless, D.D. Reeder, \\ D.L. Schumann, E.S. Smith \\ University of Wisconsin, Madison, WI 53706
}

Presented by: Robert C. Ball

\section{ABSTRACT}

The flux of prompt neutrinos from a beam dump has been measured in an experiment at the Fermi National Accelerator Laboratory (E613). Assuming that the charm production has a linear dependence on atomic number and varies as $(1-|x|)^{5} \mathrm{e}^{-2 m_{T}}$, a model dependent cross section of $27 \pm 5 \mathrm{\mu b} /$ nucleon can be derived. For neutrino energies greater than $20 \mathrm{GeV}$, the flux of electron neutrinos with respect to muon neutrinos is $0.78 \pm 0.19$. For neutrinos with energy greater than $30 \mathrm{GeV}$ and $p_{\perp}$ greater than 0.2, the flux of $\bar{v}_{\mu}$ compared to $v_{\mu}$ is $0.96 \pm 0.22$.

\section{INTRODUCTION}

Prompt neutrinos are those neutrinos produced in the creation and subsequent semi-leptonic decay of charmed particles. In producing prompt neutrinos, there is a background from non-prompt neutrinos, those from the decay of pions and kaons also produced in the target. The goal of this experiment was to maximize the prompt neutrino flux with respect to the background, and measure it as a function of the neutrino energy $E_{v}$, target a tomic number $A$, and neutrino transverse momentum PT. The relative flux of electron and muon neutrinos was also measured.

\section{EXPERIMENTAL ARRANGEMENT}

The layout of the experiment is shown schematically in FIgure 1. A beam of $400 \mathrm{GeV}$ protons was incident on a (minimum) 3 interaction

a Presently with Bell Labs, Naperville, IL

b Presently with Fermi lab, Batavia, IL 
E613 EXPERIMENT - OVERALL PLAN VIEW

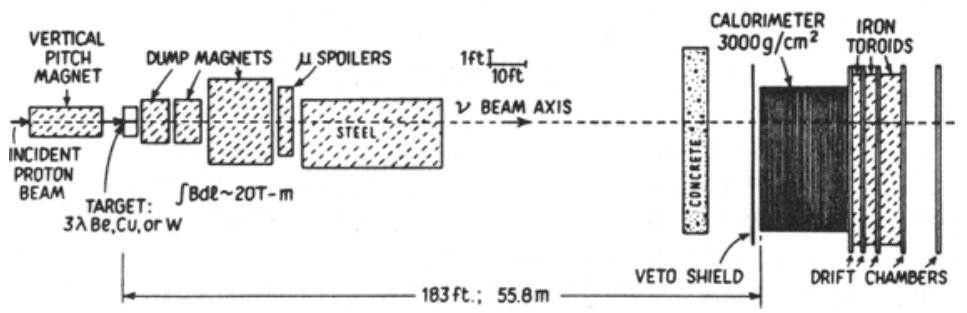

Fig. 1. Schematic 1 ayout of the experiment.

length tungsten target. The target was followed immediately by an $11 \mathrm{~m}$ magnetized iron shield which absorbed remaining strongly interacting particles and ranged out or swept aside muons aimed at the detector, which was $60 \mathrm{~m}$ from the target. The muon flux was further reduced to $3 \times 10^{5}$ per $2 \times 10^{12}$ incident protons by $11 \mathrm{~m}$ of passive iron shield.

The detector consisted of a $3000 \mathrm{~g} / \mathrm{cm}^{2}$ calorimeter followed by a muon spectrometer of iron toroids interspersed with drift chamber planes. Data collection was triggered by interactions in the calorimeter which deposited sufficient energy to exceed established thresholds. The calorimeter was segmented into 30 modules longitudinally, and each module was followed by $x$ and $y$ proportional tubes read out in the proportional mode. The modules, lead and 1 iquid scintillator sandwiches, were split vertically into 5 cells viewed by phototubes at each end. The signals from summed, overlapping sections of phototubes were used to form the trigger. The fiducial volume was confined to modules 3-25 with a transverse window $2.6 \mathrm{~m}$ wide by $1.0 \mathrm{~m} \mathrm{high}$. The calorimeter center was offset from beam center by $0.75 \mathrm{~m}$ in the long transverse dimension.

Earlier experiments at CERN $1-3$ were performed at a distance of about $900 \mathrm{~m}$ from their copper production target, and subtended a maximum angle of 2 mrad. This experiment was at a distance of $60 \mathrm{~m}$, and subtended angles up to $37 \mathrm{mrad}$. Since the non-prompt neutrinos were concentrated at smaller angles than the prompt, this helped to increase the ratio of prompt to non-prompt events over the earlier experiments' rates. Additionally we used a tungsten target which had a higher density than copper and a consequently smaller non-prompt background.

To control the neutrino background from upstream sources, a system of more than 30 beam line monitors was installed and maintained. They were calibrated by varying the beam pipe vacuum and by inserting known amounts of material into the beam. In this way, the beam 1 ine related background was determined to be 1ess than $2.0 \%$ of the full density tungsten prompt signal. 
Background from material near the target, such as air, vacuum windows, and proton monitors, was more serious but calculable. For our 1981 data period reported here, this background was $17 \%$ of the prompt signal.

\section{DATA ANALYSIS}

The technique used to extract the prompt neutrino signal was that of extrapolation. Data were collected on tunsgen targets of two different densities, nominally full density (full $\rho$ ) and 1/3-ful 1 density $(1 / 3 \rho)$. Since for the same material the non-prompt signal increases linearally with inverse density while the prompt signal remains unchanged, extrapolation to $1 / 0=0$ gives the prompt signal The near-target background correction was made by correcting the nomi nal inverse density ratio from $3: 1$ to $2.49: 1$. This is shown in Figure 2 where the total number of events normalized by the number of incident protons is plotted for the two targets.

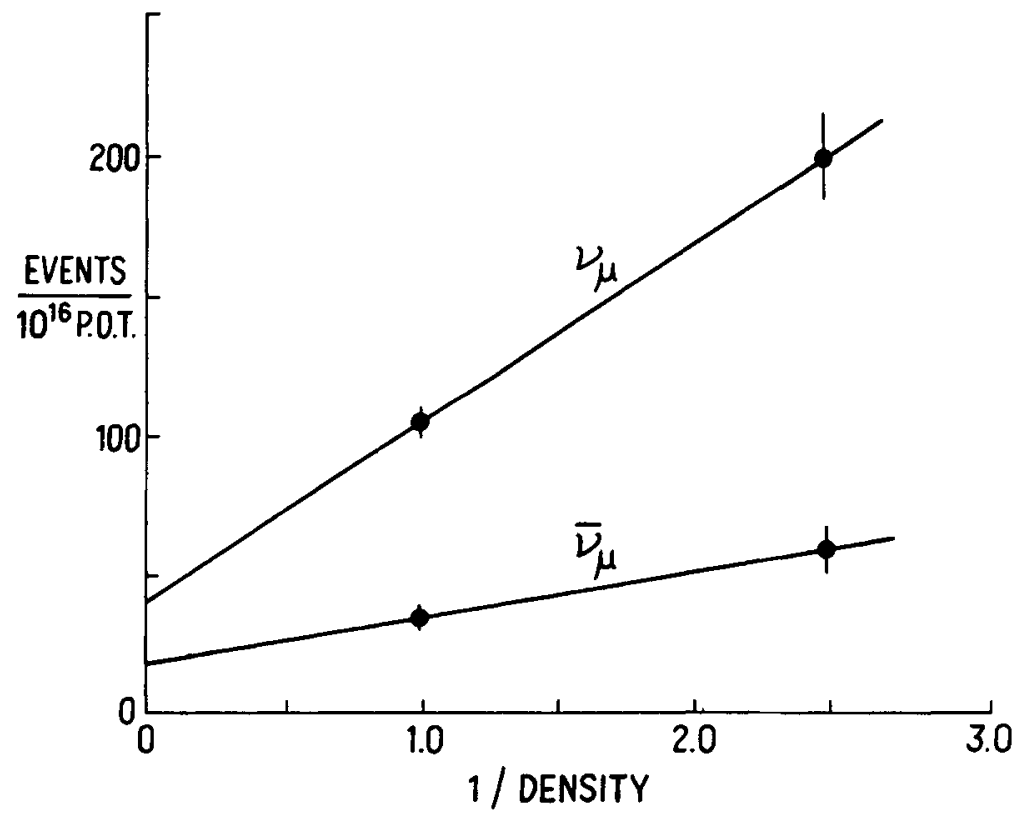

Fig. 2. Extrapolation to infinite density for $v_{\mu}$ and $\vec{v}_{\mu}$ events.

During the run period, a total of $1.6 \times 10^{17}$ protons were targeted on the two densities of tungsten. After correcting for the experiment live time $(\sim 70 \%)$ and discarding beam spills in which beam line backgrounds were high or the beam was mis-steered, a total of $6.6 \times 10^{16}$ protons incident for the full density tungsten and $1.6 \times 10^{16}$ protons for the partial density tungsten targets were kept. A total of approximately 300,000 triggers were written to magnetic tape during this time. Approximately $1 / 3$ of these triggers resulted from 
cosmic rays, and the remainder were beam related, mostly due to muons which interacted in the floor or concrete roof shielding and showered into the calorimeter. Effects of the cosmic rays were monitored by triggering with no beam on target for a time equal to that in which there was beam. These "beam-off" triggers were treated by the analysis in the same way as all other triggers. The total number of $v_{\mu}$ charged current interactions found in the fiducial volume and successful ly momentum fit was 854 . The number of $0_{\mu}$ events, which includes ve charged current (CC) events along with $v_{e}$ and $v_{\mu}$ neutral current (NC) events, based on a slightly smaller sample of protons, was 752 .

\section{RESULTS}

Three results were derived from this data, the cross section for production of DD pairs, the ratio of $\bar{v}_{\mu}$ to $v_{\mu}$ fluxes, and the ratio of $v_{e}$ to $v_{\mu}$ charged current interactions. The first result is very model dependent, the latter two are not.

To derive the cross section, the full and partial density events were divided into bins in $E_{\nu}$ and $\theta_{\nu}$ so that the prompt signal could be extracted and the acceptance of the detector could be calculated for each bin. It was then assumed 4,5 that the neutrino production varied as $(1-|x|)^{n} e^{-a r_{1}}$ where $x$ is Feynman $x, n$ is an integer, $r_{1}$ is either $p_{1}$ or $m_{1}=\left(p_{1}^{2}+m_{D}^{2}\right)^{1 / 2}$, and a is a variable describing the $r_{\perp}$ dependence. In addition, cascading of the beam protons in the target is described using a mean proton elasticity $(\varepsilon)$ of 0.3 with energy dependence of $s^{k}$ where $s=\left(2 M_{p} E_{L A B}\right)^{1 / 2}$. J. Leveille $e^{5}$ suggests the best value of $k$ is 1.3 and urges the use of $r_{\perp}=m_{\perp}$. The semileptonic branching ratio $(D \rightarrow \mu)$ is taken as the average of the $D^{+}$and $D^{\circ}$ branching fractions, $8.2 \% 6,7$. Table I shows the results reported for the various experiments $1,2,8,9$ along with their model assumptions and then corrects all results to a common model using $n=5, a=2, \varepsilon=0.3$, $k=1.3$ and $r_{\perp}=m_{1}$, which we have found gives a better fit to our data and gives a cross section for $D \bar{D}$ production of $27 \pm 5 \mu \mathrm{b}$. Figures 3 and 4 show the data plotted as functions of $E_{v}$ and $p_{1}(v)$ for $E_{v}>20 \mathrm{GeV}$ and $\theta_{v}<37 \mathrm{mrad}$. The data have been corrected for trigger efficiency, muon acceptance by the toroids, and the incomplete azimuthal acceptance for neutrinos. The flux of antineutrinos compared to the flux of neutrinos from the target, restricted to $E_{v}>30 \mathrm{GeV} / c$ and $p_{\perp}>0.2 \mathrm{GeV}$ where systematics are less severe, is found to be $0.96 \pm 0.22$.

TABLE I

Cross Sections for DD Production Quoted by Various Experiments

\begin{tabular}{|c|c|c|c|c|c|c|}
\hline \multirow[t]{2}{*}{ Group } & \multirow[t]{2}{*}{$\sigma(D \bar{D})$} & \multicolumn{4}{|c|}{$\begin{array}{c}\text { Model } \\
\text { Parameters }\end{array}$} & \multirow{2}{*}{$\begin{array}{c}\sigma(D D) \\
n=5, \quad a=2, \quad k=1.3, \\
\varepsilon=0.3, m_{\perp}\end{array}$} \\
\hline & & $n$ & a & $k$ & $\varepsilon$ & \\
\hline BEBC & $\begin{array}{l}17 \pm 4\left(v_{e}\right) \\
30 \pm 10\left(v_{u}\right)\end{array}$ & 3 & 2 & 0.5 & $2 / 3$ & $\begin{array}{l}46 \pm 17 \\
81 \pm 27\end{array}$ \\
\hline $\begin{array}{l}\text { CHARM } \\
\text { CCFRS } \\
\text { E- } 613\end{array}$ & $\begin{array}{l}19 \pm 6 \\
13 \pm 1 \\
27 \pm 5\end{array}$ & $\begin{array}{l}4 \\
3 \\
5\end{array}$ & $\begin{array}{c}2 \\
2 \\
2\left(m_{1}\right)\end{array}$ & $\begin{array}{l}-- \\
1.3 \\
1.3\end{array}$ & $\begin{array}{l}-- \\
0.3 \\
0.3\end{array}$ & $25 \pm 2\left(\begin{array}{c}29 \pm 9 \\
27 \pm 5\end{array}\right.$ \\
\hline
\end{tabular}




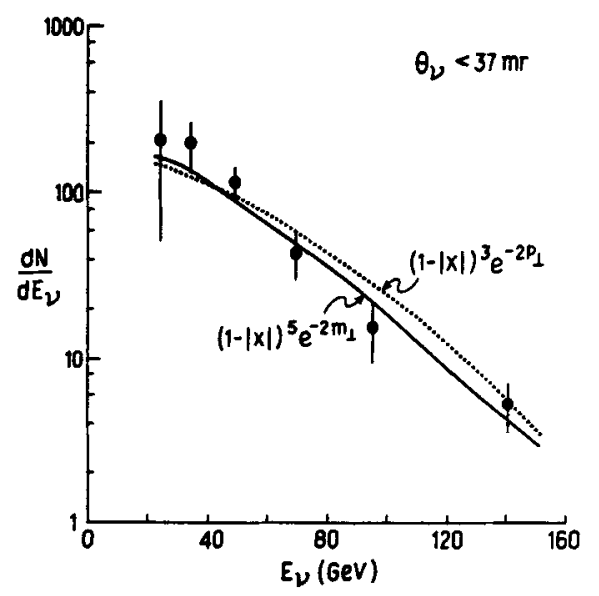

Fig. 3. Number of prompt neutrino events per $10 \mathrm{GeV}$ plotted against $E_{v}$, with the predictions of two models superimposed. The data are corrected for trigger efficiency, muon acceptance, and incomplete azimuth.

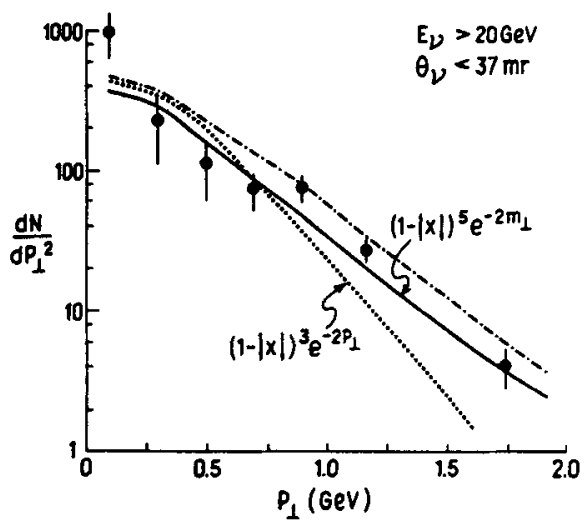

Fig. 4. Number of prompt neutrino events per $0.2 \mathrm{GeV} / \mathrm{c}$ plotted vs. $p_{\perp}$. The dot-dashed line indicates the model prediction $(n=5, a=2)$ with no energy or angul ar restrictions imposed. 
The method used to determine the ratio $R=v_{e}(C C) / \nu_{\mu}(C C)$ took advantage of the large difference in size between electromagnetic showers and hadronic showers in our calorimeter which was due to the use of $\mathrm{Pb}$ plates rather than iron or marble. Each module was 14.4 radiation lengths but only 0.5 interaction lengths. In the future, this feature will be used to make a direct separation of $v_{e}(C C)$ events from the neutral current contamination. For now it has been used to make a probabilistic detemination of the neutrino energy of the $0_{\mu}$ events (those events with no visible final state muon). Cosmic rays were then subtracted, the prompt signal was extracted, and the result was normalized to the number of incident protons. The resulting distribution included $v_{e}(C C)$ events as well as $v_{e}(N C)$ and $v_{\mu}(N C)$ events. These latter two were subtracted by using the normalized hadronic energy distribution of the $v_{\mu}(C C)$ events. The direct separation method described above gives results which agree with the method used here.

The result of this analysis is that for $E_{v}>20 \mathrm{GeV}$, the ratio $R=v_{e}(C C) / \nu_{\mu}(C C)=0.78 \pm 0.19$. For $E_{v}>20 \mathrm{GeV}$ the value $R=1.0 \pm 0.3$, and for $E_{v}>40 \mathrm{GeV}, R=1.1 \pm 0.4$. This result is shown in Figure 5 . The region where the ratio $R$ significantly deviates from unity is also the region in which the systematics, such as event finding and muon reconstruction, are most severe and can most easily distort the results.

\section{ACKNOWL EDGEMENTS}

We wish to acknowledge the efforts of the Fermilab staff and in particular the Meson Lab personnel for their efforts in our behalf. The support of the technical staff at our various institutions has been invaluable. This work was supported in part by the U.S. National Science Foundation and by the U.S. Department of Energy.

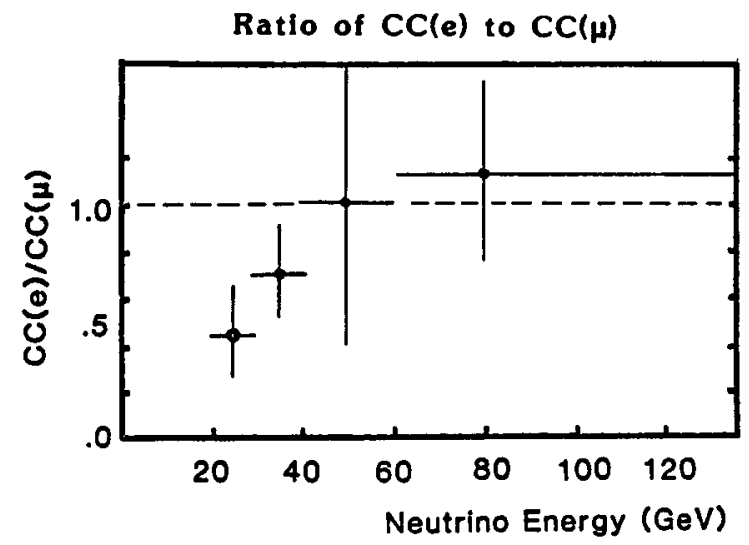

Fig. 5. The ratio of prompt $v_{e}(C C)$ over $v_{\mu}(C C)$ events vs. energy (preliminary). 


\section{REFERENCES}

1. P. Fritze et a1., Phys. Lett. 96B, 427 (1980).

2. M. Jonker et al., Phys. Lett. $\overline{96 \mathrm{~B}}, 435$ (1980).

3. H. Abramowicz et a 1., Z. Physi $\bar{k}$ 13, 179 (1982).

4. C. Michael, Proceedings of the Fourteenth Recontre de Moriond, Les Arcs (Savole), France, March, 1979, Vol. I Editions Frontieres, Oreux, France (1979), edited by J. Tran Than Van.

5. J. Leveille, University of Michigan Preprint, UM HE 82-20.

6. J.M. Feller et a 1., Phys. Rev. Letters 40, 274 (1978).

7. W. Bacino et al., Phys. Rev. Letters 43, 1073 (1979).

8. A. Bodek, Proceedings of Neutrino 182 , Balatonfüred, Hungary, June, 1982, edited by $A$. Frenkel and $L$. Jenik, p. 109.

9. A. Bodek et al., talk presented by J.L. Ritchie at the XIII International Symposium on Multiparticle Dynamics, Volendam, Netherlands, June, 1982. 